\title{
Mapping Residual Structure in Intrinsically Disordered Proteins at Residue Resolution Using Millisecond Hydrogen/Deuterium Exchange and Residue Averaging
}

\author{
Theodore R. Keppel, ${ }^{1,2}$ David D. Weis ${ }^{1}$ \\ ${ }^{1}$ Department of Chemistry, The University of Kansas, 1251 Wescoe Hall Drive, Lawrence, KS 66045, USA \\ ${ }^{2}$ Present Address: Campus Box 8069, 660 S. Euclid Ave., St. Louis, MO 63110, USA
}

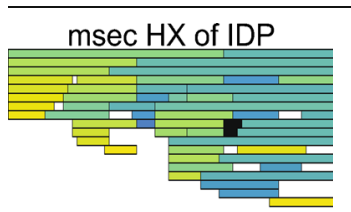

Residual helicity

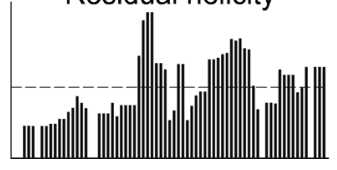

Abstract. Measurement of residual structure in intrinsically disordered proteins can provide insights into the mechanisms by which such proteins undergo coupled binding and folding. The present work describes an approach to measure residual structure in disordered proteins using millisecond hydrogen/deuterium $(H / D)$ exchange in a conventional bottom-up peptide-based workflow. We used the exchange mid-point, relative to a totally deuterated control, to quantify the rate of $H / D$ exchange in each peptide. A weighted residue-by-residue average of these midpoints was used to map the extent of residual structure at near single-residue resolution. We validated this approach both by simulating a disordered protein and experimentally using the p300 binding domain of ACTR, a model disordered protein already wellcharacterized by other approaches. Secondary structure elements mapped in the present work are in good agreement with prior nuclear magnetic resonance measurements. The new approach was somewhat limited by a loss of spatial resolution and subject to artifacts because of heterogeneities in intrinsic exchange. Approaches to correct these limitations are discussed.

Keywords: H/D exchange mass spectrometry, Intrinsically disordered protein, Residual structure

\section{Introduction}

$\mathrm{I}^{\mathrm{n}}$ ntrinsically disordered proteins contain regions that lack well-defined structure under in vivo or native in vitro conditions [1-3]. In higher organisms, roughly one-third of the expressed proteins are expected to contain disordered regions [4]. While folded proteins are essential in cellular functions such as catalysis and structure, disordered proteins play essential roles in cellular signaling and protein-protein interactions [5]. Central to their functions in protein-protein interactions is the phenomenon of coupled folding and binding, where a disordered protein becomes folded upon engagement with its binding partner [6]. Two distinct modes of binding have been proposed. One mode, known as conformational selection, proposes that the disordered protein exists as an ensemble of interconverting conformers that transiently populates folded

Electronic supplementary material The online version of this article (doi:10.1007/s13361-014-1033-6) contains supplementary material, which is available to authorized users.

Correspondence to: David Weis; e-mail: dweis@ku.edu states. Under conformational selection, the binding partner engages only with folded conformations. Alternatively, in the induced folding mode, the disordered protein first forms an encounter complex with its binding partner. It is interactions with the surface of the binding partner that lead to the folding of the disordered protein. A more detailed understanding of these modes of interaction will provide deeper insights into the principles of molecular recognition that underlie protein-protein interactions involving disordered proteins. In particular, mapping and quantifying regions of disordered proteins that are transiently structured (so-called residual structure) can support or refute the conformational selection mode of binding. Yet, obtaining measurements of residual structure remains challenging.

One technique that can provide information about residual structure is amide hydrogen/deuterium (H/D) exchange. The rate of amide $\mathrm{H} / \mathrm{D}$ exchange is highly dependent on the backbone dynamics in different regions of a protein: amides in unstructured elements exchange rapidly, whereas rigidly structured elements can exchange many orders of magnitude more slowly [7-9]. Mass spectrometry lends itself well to measurement of deuterium incorporation [10]. Previously, we and 
others have found that $\mathrm{H} / \mathrm{D}$ exchange measurements on disordered proteins are more useful when the exchange process is measured on the millisecond timescale [11-14]. In a conventional H/D exchange experiment, the relative rates of deuterium incorporation in different parts of the protein can be estimated by subjecting the deuterated protein samples to rapid proteolysis under conditions that preserve the deuterium label [15]. Still, this approach cannot match the spatial resolution achievable using nuclear magnetic resonance (NMR). For this reason, experimental [16-18] or computational [19-21] approaches that provide single residue resolution are highly desirable.

In this work, we bring together an approach that meets the need for mapping residual structure in intrinsically disordered proteins with near single-residue resolution. Our approach is built upon a simple home-built quench-flow device for millisecond H/D exchange, a model-free analysis of the peptide level H/D exchange kinetics, and a simple computational approach that takes advantage of highly overlapping peptic peptide maps. We demonstrate the feasibility of these approaches using a disordered domain of ACTR, residues 1023-1093, (activator for thyroid hormone and retinoic acid receptors or p160), hereafter referred to simply as ACTR, which binds to the co-activation domain CREB binding protein (hereafter referred to as CBP). ACTR is a nearly-random coil protein that folds upon binding to CBP [22]. While early work on ACTR suggested that the free protein was completely unstructured $[22,23]$, more recent NMR measurements have revealed that ACTR has small amounts of transient structure in the regions that become folded upon binding to CBP $[24,25]$.

\section{Experimental}

\section{Protein}

Human ACTR ${ }^{1023-1093}$ (see UniProt NCOA3_HUMAN) was co-expressed with mouse $\mathrm{CBP}^{2059-2117}$ in $E$. coli strain BL21(DE3) (Life Technologies, Grand Island, NY, USA) using a pET co-expression plasmid [22] and purified using anion exchange and size-exclusion chromatography as described previously [11].

\section{H/D Exchange Mass Spectrometry}

Millisecond amide H/D exchange labeling was carried out using a home-built quench-flow device [14]. ACTR in 10 $\mathrm{mM}$ phosphate $/ 50 \mathrm{mM}$ sodium chloride $/ \mathrm{pH} 6.9$ was labeled with a 5 -fold excess of $10 \mathrm{mM}$ phosphate $/ 50 \mathrm{mM}$ sodium chloride/pD 6.9 at $22^{\circ} \mathrm{C}$. The $\mathrm{pD}$ was calculated using the glass electrode correction for the deuterium isotope effect [26]. The labeled sample was quenched with $200 \mathrm{mM}$ sodium phosphate buffer at $\mathrm{pH} 2.6$ at a $6: 5$ volume ratio, frozen immediately with liquid nitrogen, and held at $-80^{\circ} \mathrm{C}$ until analyzed. Totally deuterated control samples were prepared manually by labeling for at least $14 \mathrm{~h}$ at $22^{\circ} \mathrm{C}$ using a 5 -fold excess of labeling buffer and then quenched as described above. Immediately prior to LC-MS analysis on a time-of-flight mass spectrometer (Agilent 6220; Santa Clara, CA, USA), individual samples were rapidly thawed by hand and injected into a refrigerated column compartment constructed in-house [27]. Following rapid proteolysis of the quenched samples in an immobilized pepsin column [28] at $0^{\circ} \mathrm{C}$, ACTR peptides were desalted using a $\mathrm{C}_{12}$ trap and separated with a water/acetonitrile gradient, as described previously [11]. All measurements were based on the analysis of three technical replicates of the labeling. Each replicate was analyzed on a separate day. The average deuterium recovery was $80 \% \pm 8 \%$ (mean \pm standard deviation), after accounting for a theoretical maximum exchange of $83.3 \%$ (1:5 dilution with $\mathrm{D}_{2} \mathrm{O}$ ).

Peptic peptides of ACTR were identified using a combination of accurate mass measurements $( \pm 10 \mathrm{ppm})$ on a time-of-flight mass spectrometer (Agilent model 6220; Santa Clara, CA, USA ) and MS/MS using collision-induced dissociation in a linear ion trap (Thermo LTQ-XL). Peptide MS/MS spectra were assigned using Proteome Discoverer (Thermo, San Jose, CA, USA) and validated by manual inspection of the assigned peaks. A set of 63 peptides was used in this analysis (see Table S1 in the Supporting Information). The peptide set was scrutinized to remove potential $y$ ion fragments of larger peptides that might be generated by insource fragmentation [29]: when co-eluting peptides shared a C-terminal cleavage site, the shorter peptides were rejected unless the N-terminal cleavage could be substantiated by another peptic peptide.

\section{Data Analysis}

Initial $\mathrm{H} / \mathrm{D}$ exchange data processing was carried out using HDExaminer (Sierra Analytics, Modesto, CA, USA). Deuterium uptake was normalized to $100 \%$ using the totally deuterated values [15]. The data were then exported to a spreadsheet for subsequent analysis. The time required to reach $50 \%$ deuteration $\left(\bar{t}_{50 \%}\right)$ was determined by linear interpolation between the two data points spanning $50 \%$ exchange. Here, the overbar indicates that the value represents the average exchange over the entire peptide. The determination of residue-resolved $t_{50 \%}$ values began with the generation of an 'exchangeability' matrix, $E$, with dimensions $i \times j$, analogous to the Kronecker delta function, illustrated schematically in Figure 1. The index $i$ denotes the individual peptides and $j$ denotes the residues in the protein. The values in this matrix, either 1 or 0 , indicate whether a given residue, $j$, in a given peptide, $i$, reports on exchange (i.e., $E_{i, j}=1$ ), or not (i.e., $E_{i, j}=0$ ). After proteolysis, back-exchange causes complete loss of deuterium at the first residue of a peptide because this position is converted from an amide to an amine. Intrinsic exchange calculations show that the second residue (i.e., the first amide deuterium in the peptide) also undergoes rapid back-exchange under quench conditions [30,31]. Thus, the first two residues of peptide $i$ and any 


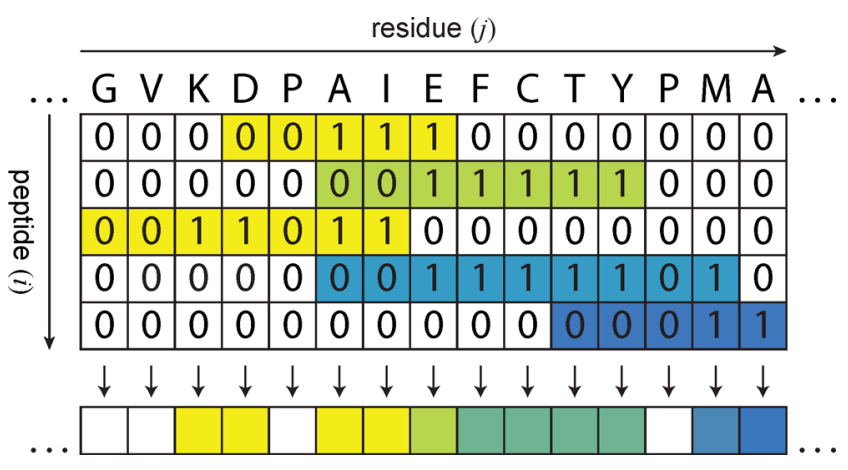

Figure 1. H/D exchange kinetics mapped onto individual amides using weighted residue averaging as described in the Experimental section. Individual peptides are denoted by colored boxes. The midpoint of exchange $\left(\bar{t}_{50 \%}\right)$ for each peptide is indicated by color with a shading scheme between fast exchange (yellow) and slow exchange (blue). The values of zero and one indicate whether a given residue $(j)$ contributes to the observed exchange in peptide $i$. The residue-averaged values at the bottom are obtained by using a weighted average of $\bar{t}_{50 \%}$ for residues assigned a value of one

proline residues were assigned $E$ values of 0 . All other residues in each peptide were assigned values of 1 . Residues that were not contained within peptide $i$ were also assigned a value of 0 . Formally,

$E_{i, j}=\left\{\begin{array}{l}0, \text { if } j<n_{i}+2 \text { or } j>c_{i} \text { or } R_{j}={ }^{\prime \prime} \mathrm{P}^{\prime \prime} \\ 1, \text { if } n_{i}+2 \leq j \leq c_{i} \text { and } R_{j} \neq{ }^{\prime \prime} \mathrm{P}^{\prime \prime}\end{array}\right.$

where $n$ and $c$ denote the $\mathrm{N}$ - and C-termini of each peptide and $R_{j}$ is the identity of the residue $j$. The peptide-averaged $\bar{t}_{50 \%}$ values were mapped uniformly onto each residue of a given peptide. The residue-resolved values of $t_{50 \%}$ were obtained from a weighted average of $\bar{t}_{50 \%}$ values from all peptides that covered a given residue, as depicted schematically in Figure 1 by the vertical arrows:

$t_{50 \%, j}=\frac{\sum_{i}\left(E_{i, j} \cdot \bar{t}_{50 \%, i} \cdot w_{i}\right)}{\sum_{i} w_{i}}$

The weighting function $w$ in Equation 2 gives more weight to peptides with fewer exchangeable residues than to peptides with larger numbers of exchangeable residues, since the shorter peptides provide better spatial resolution. Here, the weighting function was based on the inverse square of the number of exchangeable residues:

$w_{i}=\left(\sum_{i} E_{i, j}\right)^{-2}$

Equations 1 through 3 were implemented in a series of spreadsheets. (a)
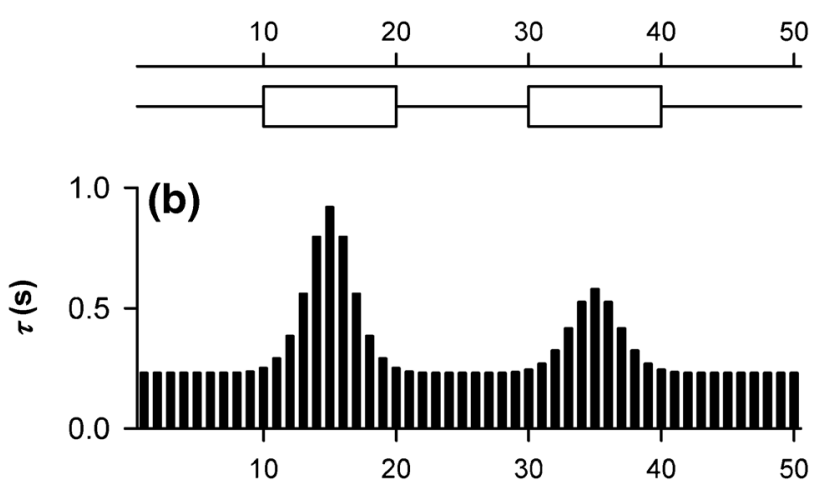

(c)

$t_{50 \%}(\mathrm{~s})$
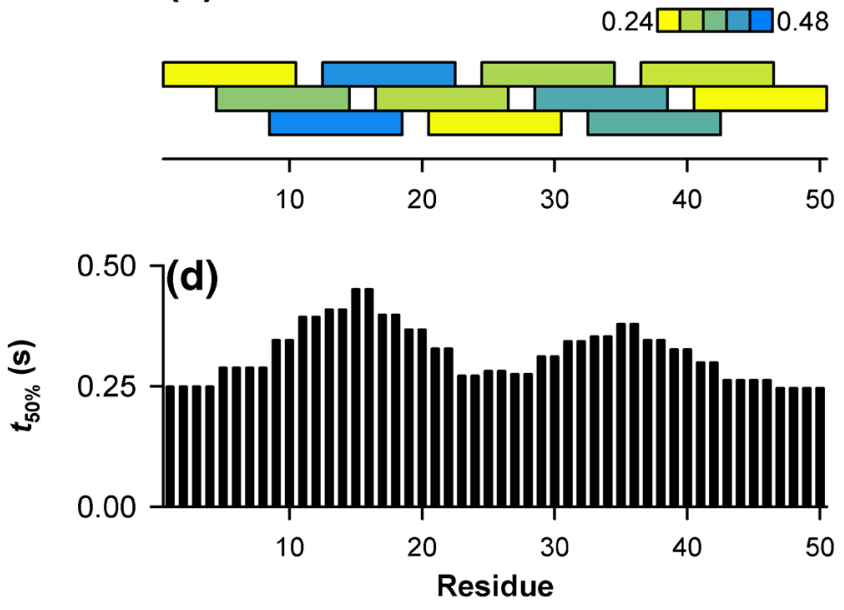

Figure 2. Residue averaging of $\bar{t}_{50 \%}$ values accurately returns the protected regions in a simulation of a weakly protected loop-helix-loop-helix-loop protein. The secondary structural elements are shown in (a). The simulated half-lives $(\tau)$ of exchange of individual residues are shown in (b). The $\bar{t}_{50 \%}$ values for overlapping, 10 residue peptides are shown in panel (c), colored as shown in the scale bar. Using residue averaging of the $\bar{t}_{50 \%}$ values, as described in the Experimental section, residue-averaged values of $t_{50 \%}$ were computed. The results are shown in (d). See text for more details

The simulation of H/D exchange used a model polypeptide composed of two 10-residue helical domains inserted between three 10-residue linker regions (see Figure 2a). The amides in the linker regions were assigned a half-life of $0.23 \mathrm{~s}$, typical of intrinsic exchange by unstructured polypeptides at $25^{\circ} \mathrm{C}$ at $\mathrm{pD} 7$ [30]. Gaussian distributions centered in the middle of each helix were used to define the half-lives of exchange in the helical regions (see Figure 2b). The slowest exchanging amides in the middles of the $\mathrm{N}$ - and C-terminal helices had half-lives $(\tau)$ of $0.92 \mathrm{~s}$ and $0.58 \mathrm{~s}$, respectively, corresponding to protection factors of 4 and 2.5, respectively. Amide $\mathrm{H} / \mathrm{D}$ exchange was simulated in a series of 10-residue peptides, each offset by four residues 
from the previous peptide, using a sum of single exponentials:

$D(t)=\sum_{j}\left(1-e^{-\frac{t}{\tau_{j}}}\right)$

where $\tau_{j}$ is the half-life of exchange of residue $j$ and $t$ is the exchange time. For simplicity, it is assumed in this model that all residues in the peptide undergo detectable $\mathrm{H} / \mathrm{D}$ exchange. In other words, there are no prolines, there is no conversion of the first amide into an amine, and the second residue does not rapidly back-exchange. The simulated deuterium uptake curves can be found in the Supplementary Information section (Figure S1). $\bar{t}_{50 \%}$ for each peptide was determined by linear interpolation. Residue-averaged $t_{50 \%}$ values were determined using Equations 1 and 2 without weighting (i.e., $w=1$ ), since all peptides were of equal length. These results are shown in Figure $2 \mathrm{c}$ and $\mathrm{d}$.

The helical propensity of ACTR was predicted using the AGADIR algorithm [32-34] available online at http:// agadir.crg.es/. ACTR disorder prediction was calculated using the PONDR-VL $\mathrm{XT}_{\mathrm{X}}$ algorithm [35] available online at http:// www.pondr.com/.

\section{Results}

In this work, we propose a novel, model-free approach to parameterize peptide-level H/D exchange kinetics and obtain residue-resolved results by taking advantage of the many overlapping peptides produced by pepsin digestion. We describe exchange using empirically-determined mid-point values, $t_{50 \%}$, which represent the time required to reach $50 \%$ deuteration relative to a totally deuterated control sample. We avoid the use of the term 'half-life' in this context since peptide-level exchange is an ill-defined multi-exponential process. We illustrate the use of this approach by defining residually-structured helical elements in the intrinsically disordered protein ACTR.

The approach to obtaining residue-resolved kinetics, described in the Experimental section, is presented schematically in Figure 1. We used a weighted average of the mid-points of exchange $\left(\bar{t}_{50 \%}\right)$ of every peptide that covers a given residue. To validate this averaging approach, we modeled exchange in a simple two-helix polypeptide in which the two helices exhibit only very weak protection against exchange (see Figure $2 \mathrm{a}$ and b). Using the model, we simulated peptide-level H/D exchange (see Supplementary Figure S1) to determine the midpoint of exchange $\left(\bar{t}_{50 \%}\right)$ by each peptide, as shown in Figure 2c. All residues, except for the first and last four, are covered by either two or three peptides. The residue-resolved midpoint data, $t_{50 \%}$, shown in Figure 2d, compare favorably with the actual individual half-lives $(\tau)$ of the amides, shown in Figure 2b. In particular, the boundaries of both helical regions are welldefined. The gradations of protection across each helix and the locations of maximum protection can also be easily identified. Finally, the difference in the relative magnitudes of protection in the two helices is also returned accurately using the peptide-averaging approach. There is some degradation in the quality of the data. Some loss of both spatial resolution and dynamic range is evident in Figure 2d. These losses arise from the blurring of the data that arises from trying to retrieve single residue resolution from overlapping 10 residue fragments. Overall, however, the results from the simulation establish the feasibility of using residue averaging of the $\bar{t}_{50 \%}$ values to achieve higher resolution for weakly protected secondary structural elements from peptide maps where most residues are covered by at least two or three peptides.

Representative deuterium uptake data from selected peptides covering the entirety of the ACTR sequence are shown in Figure 3. These results have been normalized to $100 \%$ relative to fully deuterated ACTR. As is evident in the figure, ACTR peptides exhibit a range of different amide H/D exchange kinetic profiles. The time required to reach $50 \%$ deuteration can be estimated from Figure 3 using the dashed horizontal reference line at $50 \%$. At one extreme, the $\mathrm{N}$-terminal peptide $1023-1030$ is $50 \%$ exchanged after approximately $0.2 \mathrm{~s}$ and is almost fully exchanged by $3.5 \mathrm{~s}$. In contrast, the 1051-1057 peptide takes $1.6 \mathrm{~s}$ to reach $50 \%$ and is only $\sim 65 \%$ deuterated after $3.5 \mathrm{~s}$. This represents a roughly 16 -fold difference in the rate of H/D exchange in different peptides of ACTR.

Although a variety of different approaches to parameterize deuterium incorporation kinetics have been described in the literature [15, 19-21, 36-38], peptide-level H/D exchange is inherently a multi-exponential process involving kinetics of both forward and back-exchange. It is well known that analysis of multi-exponential behavior is computationally challenging, particularly when the data are sparse [39]. To avoid these issues, we have adopted the empirical approach of interpolating the amount of time required to reach $50 \%, \bar{t}_{50 \%}$, using simple linear interpolation between the two exchange measurements that span $50 \%$. The peptide-level $\bar{t}_{50 \%}$ values that arise from this simple analysis, mapped onto the ACTR sequence, are shown in Figure 4. Two peptides (1070-1072 and 10701073), shown in black, exchange too slowly to reach 50\% exchange within the timescale of the quench-flow exchange system. These two peptides were excluded from subsequent analysis. In general, peptides from the N-terminal third of ACTR are less protected. This less protected region correlates with the 24-residue $\mathrm{N}$-terminal tail that is absent from the NMR-derived structure of the ACTR-CBP complex [22]. The most slowly-exchanging peptides, shown in blue and black, align with regions of ACTR that become helical in the complex (shown in Figure 4a).

Using this approach, we obtained residue-resolved $t_{50 \%}$ values by residue averaging the data shown in Figure 4. The results are shown in Figure $5 \mathrm{~b}$. The $t_{50 \%}$ values range from $0.23 \mathrm{~s}$ in N-terminal tail to a maximum of $1.03 \mathrm{~s}$ in helix 1 . In general, there is good correlation between residues with $t_{50 \%}$ values greater than $0.5 \mathrm{~s}$ (dashed line in Figure $5 \mathrm{~b}$ ) and the regions of ACTR that become helical in complex with CBP. However, there is less protection in residues 1058 and 1059 than in the rest of the residues in helix 1. There is also some blurring of the $\mathrm{N}$-terminal boundary of helix 2 , with $t_{50 \%}$ values 


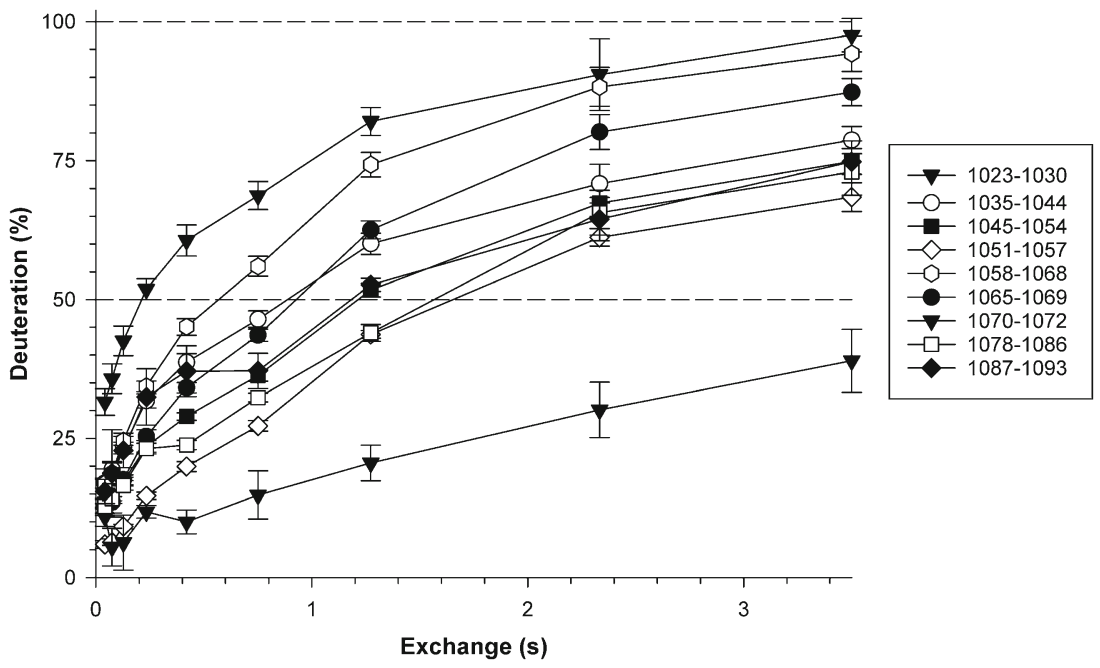

Figure 3. Representative deuterium uptake curves from ACTR peptic peptides exhibit a range of deuterium uptake kinetics; $\bar{t}_{50 \%}$ values were determined by linear interpolation (the point where each curve crosses the 50\% line). Data were normalized to $100 \%$ relative to a totally deuterated control [15]. The error bars denote the standard deviations from triplicate measurements

suggesting that this helix extends to residue 1067. It is worth noting that the author-deposited secondary structure assignment in the Protein Databank extends helix 2 to residue 1066 (see $1 \mathrm{KBH}$ ), whereas Figure 5a shows the secondary structure assignment using the DSSP algorithm [40]. Finally, in the Cterminal tail, residues 1083-1093, exchange is much slower than in the disordered $\mathrm{N}$-terminal tail. At neutral $\mathrm{pH}$, exchange at the C-terminal amide of ACTR is hindered by two carboxylate groups that repel $\mathrm{OD}^{-}$, resulting in increased protection against exchange. This factor contributes to a calculated intrinsic exchange half-life of $33 \mathrm{~s}$ for the C-terminal amide of ACTR [30]. This amide is essentially non-exchanging on the millisecond timescale, leading to some bias in the measurement of $\bar{t}_{50 \%}$ in all of the C-terminal peptides.

\section{Discussion}

There are two alternative models that describe how intrinsically disordered proteins undergo coupled binding and folding with their interaction partners. These interactions might involve either binding of a transiently-folded conformer or, alternatively, formation of an encounter complex that induces folding [2, 6]. Measuring the extent to which the isolated protein samples folded conformations in the absence of its binding partner can provide better insights into the nature of protein-protein interactions involving disordered proteins. Our goal in this work is to explore the potential for millisecond $\mathrm{H} / \mathrm{D}$ exchange and residue averaging to provide this type of information.

(a)

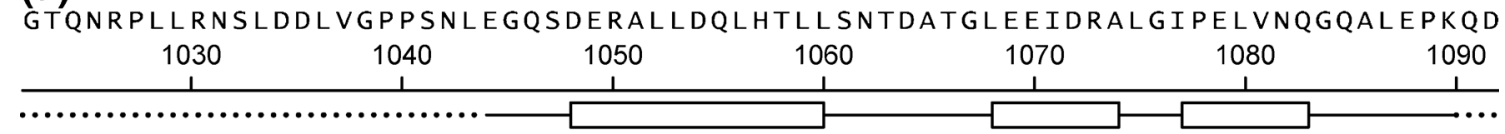

(b)

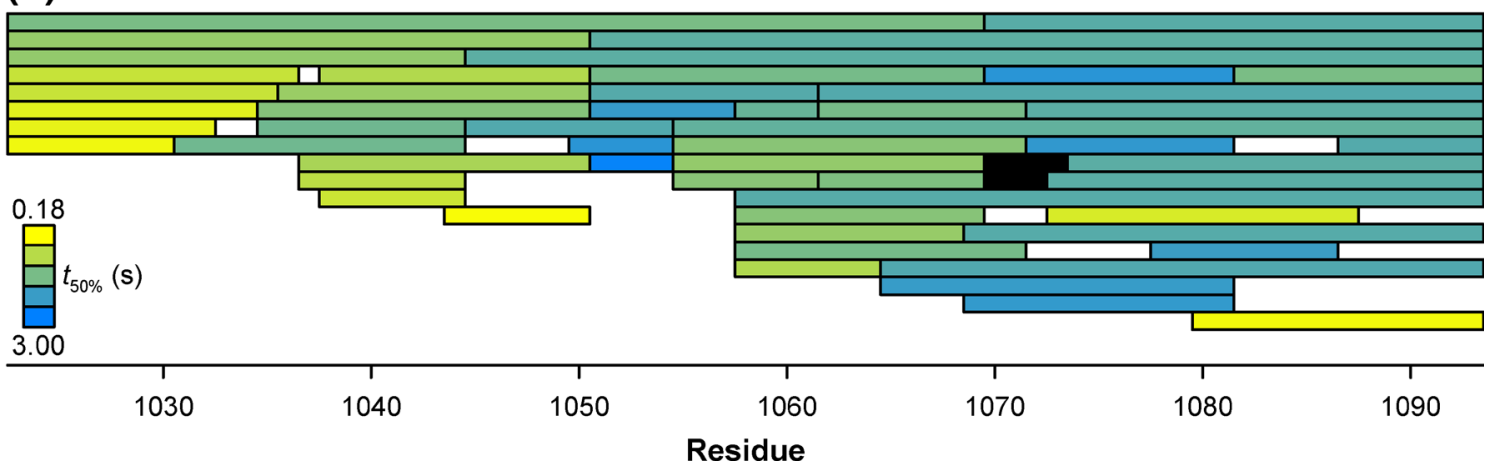

Figure 4. $\bar{t}_{50 \%}$ values (in seconds) for each peptic peptide of ACTR are aligned against the primary sequence and secondary structure (a) that ACTR forms in complex with its binding partner [22]. (b) The $\bar{t}_{50 \%}$ values are indicated by the color scale that runs from $0.18 \mathrm{~s}$ in yellow to $3 \mathrm{~s}$ in blue. The scale is logarithmic, with each box in the scale denoting a two-fold increase in $\bar{t}_{50 \%}$. Two peptides, shown in black, did not reach $50 \%$ exchange 

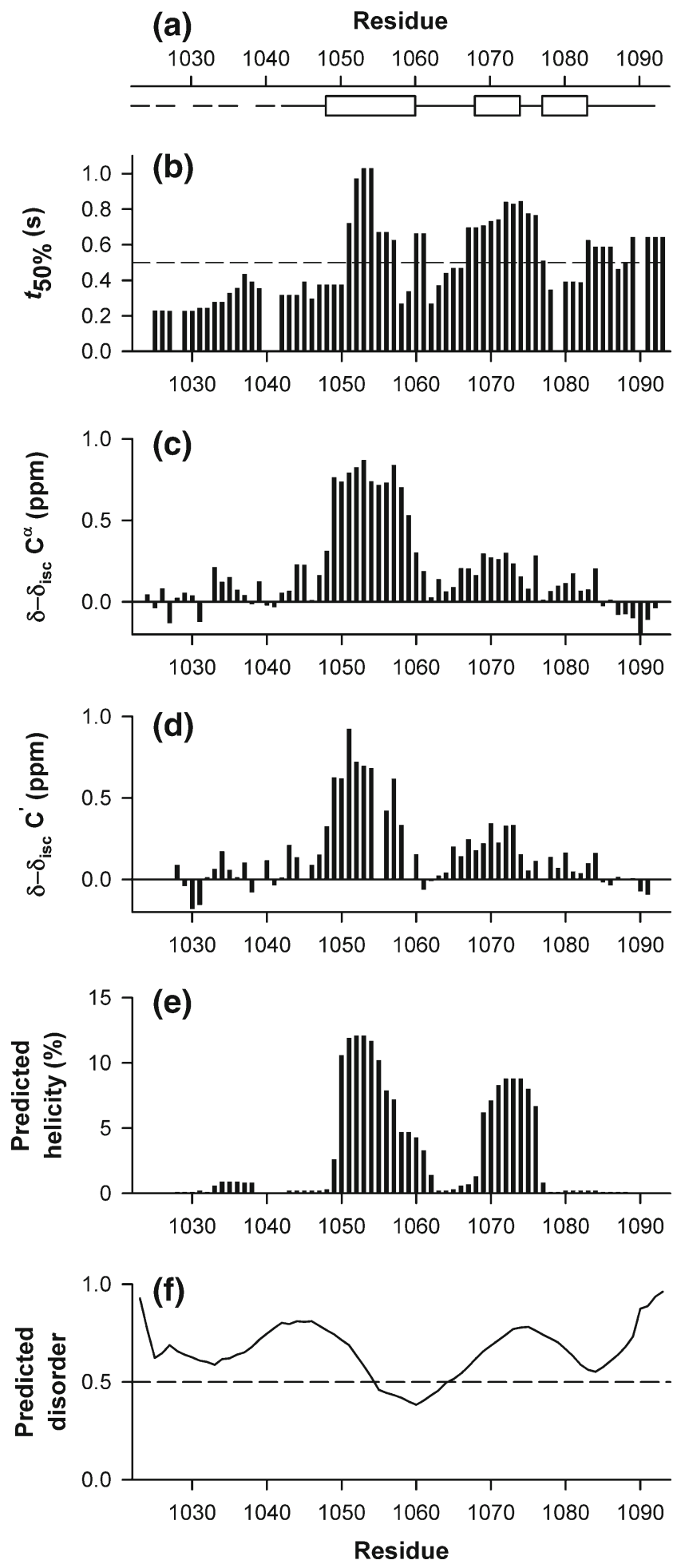

Figure 5. Residue averaged exchange can be used to measure and map residual structure in an intrinsically disordered protein. Measures of residual structure and disorder are aligned against the $\alpha$-helical secondary structure that ACTR forms in complex with CBP (a) [22]; $t_{50 \%}$ values obtained by averaging the peptide data (Figure 4) are shown in (b). Secondary chemical shift data obtained by NMR are shown in (c) and (d) [41]. A helical propensity prediction and a disorder prediction are shown in panels (e) and (f), respectively
In the case of ACTR, a small, soluble protein that does not appear to be prone to aggregation and can be easily isotopically-labeled, NMR has provided valuable insights into the extent of residual structuring [22, 23, 25, 41]. The measurement of chemical shifts for the backbone carbon atoms $\left(\mathrm{C}^{\alpha}\right.$ and $\left.\mathrm{C}^{\prime}\right)$ relative to the chemical shifts of the same protein measured in 6 M urea [24] has been used to map regions of ACTR that are transiently helical (see Figure 5c and d) [41]. Large, positive secondary chemical shifts are indicative of residual structure. Also shown in Figure 5 are helical propensity and disorder predictions for ACTR (Figure 5e and f). ACTR can serve as a useful model system to validate millisecond H/D exchange measurements.

In general, the data shown in Figure 5b are in good agreement with NMR secondary chemical shift data. Both types of measurements show that the extent of residual structuring is strongest in helix 1, in good agreement with the helical propensity prediction. The biggest discrepancy is in the helix 2 region, where $\mathrm{H} / \mathrm{D}$ exchange suggests that the degree of structuring is similar to that of helix 1. In contrast, the NMR data suggest that structuring in the helix 2 region is weaker. This discrepancy suggests several different possibilities. First, the difference may just arise as an artifact of the residue averaging process itself: there are fewer small peptides in the helix 2 region, so the exchange kinetics may not be as well-localized. A second possibility is that there is no simple linear correlation between $\mathrm{H} / \mathrm{D}$ exchange kinetics and secondary chemical shift. Finally, the data obtained from NMR and H/D exchange may report on different, but related, biophysical properties of polypeptides. Although H/D exchange kinetics are related to dynamic motions of the peptide backbone $[7,8,42]$, the secondary chemical shifts are sensitive to the extent to which the polypeptide backbone favors particular dihedral angles [43, 44]. Hence, the naïve expectation that there would be a linear correspondence between $t_{50 \%}$ and secondary chemical shift is unlikely. Interestingly, both the NMR and H/D exchange measurements suggest that there is little or no residual structure in the helix 3 region. This observation is also in good agreement with the helical propensity prediction. All of these observations imply that folding in helix 3 requires interaction with the surface of CBP [22]. Finally, we note some slight evidence of protection in the 1030-1040 region that seems to correlate with limited helical propensity and, possibly, positive secondary chemical shifts for the $\mathrm{C}^{\alpha}$ carbons. In contrast to the generally good agreement between H/D exchange, NMR, and helical propensity, there is little correlation between H/D exchange and the PONDR disorder prediction. PONDR scores range from zero for regions with a high likelihood of being ordered to one for regions with a high likelihood of being disordered. Figure $5 \mathrm{f}$ shows the disorder prediction for ACTR. Although the disorder prediction correctly shows a low likelihood for order in all regions of ACTR, the lowest PONDR scores do not align with the helical regions of ACTR.

Although there is generally good correlation between the various measures and predictions of residual secondary structure, the comparison does reveal some limitations of the 
millisecond H/D exchange approach. In general, even when weighted averaging is used, there is some degradation of spatial resolution. This is not surprising: it would be unreasonable to expect recovery of truly residue-resolved exchange from peptides that span many residues no matter how many overlapping peptides are combined. Despite the use of a weighted average, it is still possible that a small peptide could be 'overruled' by a large number of longer peptides, especially if the longer peptides cross a structured/unstructured boundary in the primary sequence. In other words, a short protected region might go undetected if the majority of peptides covering the region are long and cover adjacent unprotected regions. The averaging process could also be subject to bias either from unfavorable peptide coverage or selective assembly (i.e., cherry picking) of the peptide map. It is also important to exclude from the peptide map any in-source $y$-ion fragments [45] that might appear to be authentic proteolytic peptides.

Another issue that can arise is that intrinsic exchange, normally thought of as uniform and 'fast', can in fact be rather heterogeneous. We have already noted in the Results section that exchange by the $\mathrm{C}$-terminal aspartate amide is abnormally slow. This effect arises because of the electrostatic repulsion between the carboxylates and the deuteroxide ion [30]. Exchange will always be slow at the C-terminus, but especially slow when a second carboxylate (aspartate or glutamate) is present. Another well-known effect is steric protection of the amide by leucine, isoleucine, and valine side chains [30]. This effect is particularly evident in two ACTR peptides, EEI (10701072) and EEID (1070-1073), shown in black in Figure 4. After back-exchange, EEI has only a single, slowlyexchanging amide. In EEID, the slowly-exchanging isoleucine is further protected by the adjacent carboxylate groups. These two peptides were the only ones that did not reach $50 \%$ exchange by $3.5 \mathrm{~s}$ (see Figure 3). For well-folded proteins, these subtle intrinsic exchange effects become less important because exchange can be many orders of magnitude slower than the intrinsic rate. For intrinsically disordered proteins, however, analysis of $\mathrm{H} / \mathrm{D}$ exchange kinetics may require some sort of normalization that takes these effects into account. An alternative approach might be to measure millisecond H/D exchange relative to exchange in a high concentration of deuterated denaturant such as $d_{4}$-urea. Regardless of the relative magnitude of intrinsic exchange for a given peptide, a peptide that shows faster exchange in the presence of urea would be indicative of residual structure.

The apparently residue-resolved exchange kinetics suggested to us that it might be possible to extract residueresolved protection factors (i.e., the ratio of intrinsic to observed rate of exchange). We attempted this by first calculating peptide-level $\bar{t}_{50 \%}$ using intrinsic exchange [30] and then determining the corresponding residue resolved $t_{50 \%}$ values. Here, the results were disappointing. Many residues appeared to exchange somewhat faster than predicted on the basis of intrinsic exchange (results not shown). Indeed, many of the peptides in the intrinsically disordered protein $\alpha$-synuclein have also been shown to exchange more rapidly than expected on the basis of intrinsic exchange calculations [46]. Rand et al. also observed peptide $\mathrm{D} / \mathrm{H}$ back-exchange kinetics that were faster than predicted on the basis of intrinsic exchange [47]. These observations suggests that the intrinsic exchange calculations, based on model peptides [30,31], may not be sufficiently accurate for polypeptides that are nearly unstructured.

The highly overlapping nature of the peptic peptide map shown in Figure 4 offers the promise that H/D exchange might be sub-localized by application of a subtractive approach [19]. Recent work, however, suggests that overlapping peptides can exhibit dramatic differences in the level of back-exchange, rendering a subtractive approach based on the magnitude of exchange unreliable [48]. Instead, our approach relies on the use of $\bar{t}_{50 \%}$ to parameterize the rate, rather than the magnitude, of exchange. Our approach should be much less susceptible to back-exchange artifacts. An additional advantage of the use of $\bar{t}_{50 \%}$ is that it is empirical and model-free: no assumptions are made about the functional form of the exchange kinetics. This means the approach applies equally well to any $\mathrm{H} / \mathrm{D}$ exchange data. One possible limitation would be for peptides that exhibit H/D exchange kinetics that appear to be bi-exponential.

\section{Conclusions}

In this work, we have described an approach to mapping residual secondary structure in an intrinsically disordered protein using peptide-level millisecond $\mathrm{H} / \mathrm{D}$ exchange combined with a residue averaging algorithm to approach single-residue resolution. Although the spatial resolution is somewhat less than what can be achieved by NMR, H/D exchange mass spectrometry does not require incorporation of ${ }^{13} \mathrm{C}$ and ${ }^{15} \mathrm{~N}$ into the protein, and the approach retains the advantages of working with smaller amounts of much larger proteins. Compensation for heterogeneity of intrinsic exchange may further improve the interpretation of the results. We expect that the residue averaging approach can also be applied to conventional timescale H/D exchange data on well-folded proteins. Efforts in this area are on-going in our lab.

\section{Acknowledgments}

The authors thank Professor Peter Wright (Scripps Research Institute, La Jolla, CA) for the ACTR expression plasmid, and Dr. Magnus Kjaergaard for providing the ACTR secondary chemical shift data [41] in tabular form. Financial support for this research from a National Science Foundation CAREER award to D.D.W. (MCB-1149538) is also gratefully acknowledged.

\section{References}

1. Dunker, A.K., Brown, C.J., Lawson, J.D., Iakoucheva, L.M., Obradovic, Z.: Intrinsic disorder and protein function. Biochemistry 41, 6573-6582 (2002)

2. Dyson, H.J., Wright, P.E.: Intrinsically unstructured proteins and their functions. Nat. Rev. Mol. Cell Biol. 6, 197-208 (2005)

3. Uversky, V.N., Oldfield, C.J., Dunker, A.K.: Intrinsically disordered proteins in human diseases: introducing the D-2 concept. Annu. Rev. Biophys. 37, 215-246 (2008) 
4. Liu, J., Faeder, J.R., Camacho, C.J.: Toward a quantitative theory of intrinsically disordered proteins and their function. Proc. Natl. Acad. Sci. U. S. A. 106, 19819-19823 (2009)

5. Xie, H., Vucetic, S., Iakoucheva, L.M., Oldfield, C.J., Dunker, A.K., Uversky, V.N., Obradovic, Z.: Functional anthology of intrinsic disorder. 1. Biological processes and functions of proteins with long disordered regions. J. Proteome Res. 6, 1882-1898 (2007)

6. Wright, P.E., Dyson, H.J.: Linking folding and binding. Curr. Opin. Struct. Biol. 19, 31-38 (2009)

7. Hvidt, A., Nielsen, S.O.: Hydrogen exchange in proteins. Adv. Protein Chem. 21, 287-385 (1966)

8. Englander, S.W., Kallenbach, N.R.: Hydrogen exchange and structural dynamics of proteins and nucleic acids. Q. Rev. Biophys. 16, 521-655 (1983)

9. Konermann, L., Pan, J., Liu, Y.-H.: Hydrogen exchange mass spectrometry for studying protein structure and dynamics. Chem. Soc. Rev. 40, 1224 $1234(2011)$

10. Engen, J.R.: Analysis of protein conformation and dynamics by hydrogen/ deuterium exchange MS. Anal. Chem. 81, 7870-7875 (2009)

11. Keppel, T.R., Howard, B.A., Weis, D.D.: Mapping unstructured regions and synergistic folding in intrinsically disordered proteins with amide H/D exchange mass spectrometry. Biochemistry 50, 8722-8732 (2011)

12. Rob, T., Liuni, P., Gill, P.K., Zhu, S., Balachandran, N., Berti, P.J., Wilson, D.J.: Measuring dynamics in weakly structured regions of proteins using microfluidics-enabled subsecond H/D exchange mass spectrometry. Anal. Chem. 84, 3771-3779 (2012)

13. Goswami, D., Devarakonda, S., Chalmers M., Pascal, B., Spiegelman, B., Griffin, P.: Time window expansion for HDX analysis of an intrinsically disordered protein. J. Am. Soc. Mass Spectrom. 24, 1584-1592 (2013)

14. Keppel, T.R., Weis, D.D.: Analysis of disordered proteins using a simple apparatus for millisecond quench-flow H/D exchange. Anal. Chem. 85, 5161-5168 (2013)

15. Zhang, Z., Smith, D.L.: Determination of amide hydrogen exchange by mass spectrometry: a new tool for protein structure elucidation. Protein Sci. 2, 522-531 (1993)

16. Pan, J., Han, J., Borchers, C.H., Konermann, L.: Electron Capture dissociation of electrosprayed protein ions for spatially resolved hydrogen exchange measurements. J. Am. Chem. Soc. 130, 11574-11575 (2008)

17. Rand, K.D., Zehl, M., Jørgensen, T.J.D.: Measuring the hydrogen/ deuterium exchange of proteins at high spatial resolution by mass spectrometry: overcoming gas-phase hydrogen/deuterium scrambling. Acc. Chem. Res. 10, 3018-3027 (2014)

18. Abzalimov, R.R., Kaplan, D.A., Easterling, M.L., Kaltashov, I.A.: Protein conformations can be probed in top-down HDX MS experiments utilizing electron transfer dissociation of protein ions without hydrogen scrambling. J. Am. Soc. Mass Spectrom. 20, 1514-1517 (2009)

19. Resing, K.A., Hoofnagle, A.N., Ahn, N.G.: Modeling deuterium exchange behavior of ERK2 using pepsin mapping to probe secondary structure. J. Am. Soc. Mass Spectrom. 10, 685-702 (1999)

20. Kan, Z.Y., Walters, B.T., Mayne, L., Englander, S.W.: Protein hydrogen exchange at residue resolution by proteolytic fragmentation mass spectrometry analysis. Proc. Natl. Acad. Sci. U. S. A. 110, 16438-16443 (2013)

21. Zhang, Z., Zhang, A., Xiao, G.: Improved protein hydrogen/deuterium exchange mass spectrometry platform with fully automated data processing. Anal. Chem. 84, 4942-4949 (2012)

22. Demarest, S.J., Martinez-Yamout, M., Chung, J., Chen, H., Xu, W., Dyson, H.J., Evans, R.M., Wright, P.E.: Mutual synergistic folding in recruitment of CBP/p300 by p160 nuclear receptor coactivators. Nature $415,549-553$ (2002)

23. Ebert, M.-O., Bae, S.-H., Dyson, H.J., Wright, P.E.: NMR relaxation study of the complex formed between CBP and the activation domain of the nuclear hormone receptor coactivator ACTR. Biochemistry 47, 1299-1308 (2008)

24. Kjaergaard, M., Brander, S., Poulsen, F.: Random coil chemical shift for intrinsically disordered proteins: effects of temperature and $\mathrm{pH}$. J. Biomolec. NMR 49, 139-149 (2011)

25. Iešmantavičius, V., Jensen, M.R., Ozenne, V., Blackledge, M., Poulsen, F.M., Kjaergaard, M.: Modulation of the Intrinsic helix propensity of an intrinsically disordered protein reveals long-range helix-helix interactions. J. Am. Chem. Soc. 135, 10155-10163 (2013)
26. Glasoe, P.K., Long, F.A.: Use of glass electrodes to measure acidities in deuterium oxide. J. Phys. Chem. 64, 188-190 (1960)

27. Keppel, T.R., Jacques, M.E., Young, R.W., Ratzlaff, K.L., Weis, D.D.: An efficient and inexpensive refrigerated LC system for H/D exchange mass spectrometry. J. Am. Soc. Mass Spectrom. 22, 1472-1476 (2011)

28. Wang, L., Pan, H., Smith, D.L.: Hydrogen exchange-mass spectrometry: optimization of digestion conditions. Mol. Cell. Proteomics. 1, 132-138 (2002)

29. Kim, J.-S., Monroe, M.E., Camp, D.G., Smith, R.D., Qian, W.-J.: In-source fragmentation and the sources of partially tryptic peptides in shotgun proteomics. J. Proteome Res. 12, 910-916 (2013)

30. Bai, Y., Milne, J.S., Mayne, L., Englander, S.W.: Primary structure effects on peptide group hydrogen exchange. Proteins 17, 75-86 (1993)

31. Connelly, G.P., Bai, Y., Jeng, M.-F., Englander, S.W.: Isotope effects in peptide group hydrogen exchange. Proteins 17, 87-92 (1993)

32. Muñoz, V., Serrano, L.: Elucidating the folding problem of helical peptides using empirical parameters. Nat. Struct. Biol. 6, 399-409 (1994)

33. Muñoz, V., Serrano, L.: Elucidating the folding problem of helical peptides using empirical parameters. II. Helix macrodipole effects and rational modification of the helical content of natural peptides. J. Mol. Biol. 245, 275-296 (1995)

34. Muñoz, V., Serrano, L.: Elucidating the folding problem of helical peptides using empirical parameters. III. Temperature and $\mathrm{pH}$ dependence. J. Mol. Biol. 245, 297-308 (1994)

35. Romero, P., Obradovic, Z., Li, X., Garner, E.C., Brown, C.J., Dunker, A.K.: Sequence complexity of disordered protein. Proteins 42, 38-48 (2001)

36. Zhang, Z.Q., Li, W.Q., Logan, T.M., Li, M., Marshall, A.G.: Human recombinant $[\mathrm{C} 22 \mathrm{~A}]$ FK506-binding protein amide hydrogen exchange rates from mass spectrometry match and extend those from NMR. Protein Sci. 6, 2203-2217 (1997)

37. Althaus, E., Canzar, S., Ehrler, C., Emmett, M., Karrenbauer, A., Marshall, A., Meyer-Base, A., Tipton, J., Zhang, H.-M.: Computing H/D-exchange rates of single residues from data of peptic fragments. BMC Bioinforma 11, 424 (2010)

38. Chetty, P.S., Mayne, L., Lund-Katz, S., Stranz, D., Englander, S.W., Phillips, M.C.: Helical structure and stability in human apolipoprotein A-I by hydrogen exchange and mass spectrometry. Proc. Natl. Acad. Sci. U. S. A. 106, 19005-19010 (2009)

39. Brown, R.J.S.: Information available and unavailable from multiexponential relaxation data. J. Magn. Reson. 82, 539-561 (1989)

40. Kabsch, W., Sander, C.: Dictionary of protein secondary structure: pattern recognition of hydrogen-bonded and geometrical features. Biopolymers 22, 2577-2637 (1983)

41. Kjaergaard, M., Nørholm, A., Hendus-Altenburger, R., Pedersen, S.F., Poulsen, F.M., Kragelund, B.B.: Temperature-dependent structural changes in intrinsically disordered proteins: formation of $\alpha$ helices or loss of polyproline II? Protein Sci. 19, 1555-1564 (2010)

42. Woodward, C.K., Hilton, B.D.: Hydrogen isotope exchange kinetics of single protons in bovine pancreatic trypsin inhibitor. Biophys. J. 32, 561$575(1980)$

43. Wishart, D., Sykes, B.: The ${ }^{13} \mathrm{C}$ chemical-shift index: a simple method for the identification of protein secondary structure using ${ }^{13} \mathrm{C}$ chemical-shift data. J. Biomol. NMR 4, 171-180 (1994)

44. Spera, S., Bax, A.: Empirical correlation between protein backbone conformation and C.alpha. and C.beta. 13C nuclear magnetic resonance chemical shifts. J. Am. Chem. Soc. 113, 5490-5492 (1991)

45. Kim, J.-S., Monroe, M.E., Camp, D.G., Smith, R.D., Qian, W.-J.: In-source fragmentation and the sources of partially tryptic peptides in shotgun proteomics. J. Proteome Res. 12, 910-916 (2012)

46. Del Mar, C., Greenbaum, E.A., Mayne, L., Englander, S.W., Woods, V.L.: Structure and properties of $\alpha$-synuclein and other amyloids determined at the amino acid level. Proc. Natl. Acad. Sci. U. S. A. 102, 15477-15482 (2005)

47. Rand, K.D., Lund, F.W., Amon, S., Jørgensen, T.J.D.: Investigation of amide hydrogen back-exchange in Asp and His repeats measured by hydrogen $(1 \mathrm{H} / 2 \mathrm{H})$ exchange mass spectrometry. Int. J. Mass Spectrom. 302, 110-115 (2011)

48. Sheff, J., Rey, M., Schriemer, D.: Peptide-column interactions and their influence on back exchange rates in hydrogen/deuterium exchange-MS. J. Am. Soc. Mass Spectrom. 24, 1006-1015 (2013) 